\title{
DE L'ENCADREMENT JURIDIQUE DES RELATIONS PUBLIQUES INTERNATIONALES ENTRE LA RDC ET LA BELGIQUE
}

\author{
Par MUNDALA MUNDALA Dieudonné*
}

\section{INTRODUCTION}

Du fait de l'histoire, la Belgique a été la puissance colonisatrice de la République Démocratique du Congo ${ }^{1}$ et le système d'administration publique mis en place pour gouverner cette dernière par la métropole fut qualifié de paternaliste. Allusion était faite à une prise en charge totale de la colonie par le colonisateur sans se soucier de les former afin qu'ils acquièrent la maturité nécessaire pour leur auto prise en charge.

Mais aujourd'hui, environ cinquante-cinq ans après l'accession de la République Démocratique du Congo à la souveraineté tant nationale qu'internationale, le bilan que nous pouvons dresser à propos des relations publiques internationales entre les deux pays reste mitigé. L'on peut même oser dire que beaucoup de choses n'ont pas changé. La Belgique estime toujours avoir l'obligation d'accompagner son ancienne colonie dans tous les processus, y compris même l'influence dans sa politique extérieure. Il s'avère alors impérieux de recadrer juridiquement ces relations publiques internationales entre ces deux pays. C'est de la sorte que cette étude aura pour visée d'examiner le type des relations publiques internationales existant entre la Belgique et la République Démocratique du Congo. En clair, il s'agira de vérifier si ces relations qui sont par ailleurs historiques, peuvent être basées sur certains accords bilatéraux et si elles ont été conclues sur base du principe de l'égalité souveraine des Etats. En outre, il sied d'examiner la nature de ces accords de coopération bilatérale entre les deux pays.

D'emblée, les relations publiques internationales entre les deux pays sont à entendre comme celles tissées sur la base de l'aide publique au développement de l'ancienne colonie devenue depuis 1960 indépendante. Cependant,le même questionnement persiste, celui de savoir si ces relations se fondent également sur la base du respect mutuel entre les deux Etats ou bien elles seraient encore teintées de paternalisme au point qu'elles frisent une ingérence de la Belgique dans les affaires intérieures d'un Etat souverain, en l'occurrence la République Démocratique du Congo.

En effet, on entend par coopération internationale Belge, les actions et contributions de l'Etat belge en matière de coopération bilatérale directe, multilatérale ou bilatérale indirecte $^{2}$. Conformément à cette même loi, les pays partenaires de la Belgique sont des pays

* Assistant à la Faculté de Droit de l'Université de Lubumbashi.

1 Ce système devrait prendre fin au 30 juin 1960.

2 Art. 2 de la loi relative à la coopération internationale Belge du 25 mai 1999. 
considérés comme en voie de développement par le comité d'aide au développement, de l'organisation de la coopération et de développement économique. Tel est le cas de la République Démocratique du Congo au sein de laquelle les programmes ou projets sont financés par l'Etat belge, sur base d'une convention entre les deux pays. Dans le cadre des relations publiques internationales entre la Belgique et la RDC, plusieurs accords bilatéraux touchant à plusieurs domaines ou secteurs ont été signés. L'illustrations est faite en parlant de la convention bilatérale de sécurité sociale, la convention fiscale, accord bilatéral aérien entre la Belgique et la République Démocratique du Congo et du 16 septembre 2011 au 20 mars 2013, Kinshasa et Bruxelles venaient de signer 8 conventions spécifiques. Vu la différence du niveau économique et technique de deux pays en présence, il ne semble pas vrai que ces deux pays aient traité sur la base égalitaire. Il s'agit des relations publiques qui couvrent l'assistance belge dans plusieurs domaines en vue de relever tant soit peu le niveau de son ancienne colonie.

Dans le cadre de cette monographie axée sur l'encadrement juridique des relations publiques internationales belgo-congolaises, notre problématique est celle de connaitre le degré d'accompagnement de la politique extérieure belge au Congo et quels en sont les secteurs prioritaires? Autrement dit, la coopération belgo-congolaise s'oriente vers quels secteurs afin de déboucher à l'expansion de la RDC?

Et comment se présente la nature des accords bilatéraux conclus par ces deux nations?

En guise d'hypothèse, il sied d'affirmer que dès par l'histoire, ces deux pays tissent des relations très proches au point où la Belgique arrache en priorité au niveau de l'espace Schengen, la quasi-totalité des marchés publics relatifs à la RDC. En fait, les deux pays ont signés plus de 8 accords bilatéraux qui visent le développement de la RDC. Connaissant le rôle stratégique de la Belgique au sein de l'Union Européenne, nous estimons que les relations publiques internationales entre la RDC et la Belgique ne devraient que faciliter au partenaire Congolais l'accès aux marchés de l'Union Européenne. Si jusque-là, tel n'est pas le cas, il y'a lieu de vérifier ou d'examiner la nature des contrats bilatéraux signés entre les deux Etats. Quand l'on épingle le secteur du transport aérien qui a déjà fait l'objet d'un accord belgo-congolais, pourquoi jusque aujourd'hui le secteur de l'aviation civile congolaise ne s'est pas encore perfectionné afin que les aéronefs congolais soient autorisés à sillonner l'espace Européen? Nous estimons que l'encadrement juridique de cette Coopération belgocongolaise ainsi que le respect de cet accord bilatéral existant déjà, permettrait de concourir à l'amélioration de ces relations bilatérales et favoriserait l'expansion du Congo.

En effet, à l'heure des indépendances africaines en 1960, l'espoir de réduire les disparités entre les pays industrialisés et les pays en voie de développement était immense : les Nations Unies déclarèrent les années 1960-1970 : la décennie de développement. La coopération entre la Belgique et la RDC a commencé bien au-delà de cette période du fait des liens historiques de la colonisation. Mais rien ne permet aujourd'hui d'affirmer le décollage de la RDC tant que persiste cette disparité entre les pays industrialisés, représentés ici par la Belgique et la RDC qui, plus de cinquante ans après son accession à la souveraineté internationale, connaît du recul si pas du statu quo dans son développement. A qui incombe la 
faute? Est- ces les accords qui sont mal négociés? Il est à priori plausible qu'il s'agisse d'un problème d'hommes, et donc d'animateur.

L'intérêt de cette étude réside dans le fait que la RDC qui se trouve jusque-là dans le bloc des pays en développement, a besoin de trouver des voies de sortie pour son expansion. Et pourtant les relations belgo-congolaises sont un atout majeur pour son émergence, vu le rôle et la place stratégiques de Bruxelles au sein de l'Union Européenne et même dans le concert des nations. Pourquoi plus de cinquante ans après l'indépendance du Congo, c'est à peine que s'installe l'industrie pharmaceutique et le pays manque même une compagnie nationale d'aviation. Il n'existe pas une seule usine de montage des voitures et certaines industries minières coloniales ou textiles(tel que la cotonnière) ont fermé et sont tombées en faillite.

Dans les lignes qui suivent, nous parlerons d'abord de l'aide publique belge au développement, ensuite des secteurs prioritaires ciblés lors du sommet d'avril 2009 et enfin de la nature de la coopération belgo-congolaise.

\section{A. De l'aide publique belge au développement de la RDC.}

$\mathrm{Au}$ sortir de la seconde guerre mondiale en 1945, les pays fondateurs des Nations Unies, dont la Belgique, prirent l'engagement de favoriser le progrès économique et social de tous les peuples. A cet effet, les organismes internationaux spécialisés sont créés, des fonds récoltés, les colonies sont en voie d'émancipation. En 1957, la communauté économique européenne institue un fonds Européen de développement. En 1960, à l'heure des indépendances africaines, l'espoir de réduire les disparités entre les pays industrialisés et les pays en voie de développement est immense : les Nations Unies déclarent les années 1960-1970 : la décennie du développement. La Belgique étant un Etat qui a été créé dans le but de jouer le rôle d'arbitre parmi les grandes puissances, se devrait d'occuper une place très stratégique et elle est actuellement la capitale de l'Union Européenne. Sa responsabilité pour le développement de la RDC est vraiment historique à cause de cette indépendance brusquée, non préparée, accordée à sa colonie.

En 2000, à Monterrey au Mexique, le constat de la communauté internationale est amer : sous-alimentation, regain des pandémies, analphabétisme, surendettement, mauvaise gouvernance sont le lot de millions d'individus dans des nombreux pays, surtout africains.Les Nations Unies se donnent alors des objectifs à atteindre en 2015, identiques pour la plupart à ceux que l'on s'était déjà donné quarante ans plus tôt. La question se pose alors : l'aide au développement n'a -t'elle servi à rien? Et qu'a-t-on fait de ces fonds et budgets, destinés à éliminer la pauvreté, l'injustice et les inégalités dans le monde ${ }^{3}$. Il est important de comprendre la base de la politique extérieure belge afin de mieux appréhender la nature des relations publiques internationales entre les deux pays.

3 PATRICK DEVELTERE et ARISTIDE MICHEL, Chronique d'un demi-siècle de coopération belge au développement, La coopération Belge au développement, Service de communication, Bruxelles, www.diplomatie.be, www.dg-d.be, consulté le 22janvier 2015, vers 11h24'. 


\section{La Politique Belge d'aide au développement}

Historiquement, la nécessité d'une reconstruction matérielle et morale ainsi que l'accélération du mouvement de décolonisation ont suscité un élan de solidarité qui a connu plusieurs modes d'actions. La politique belge reste marquée par le traumatisme d'une indépendance mal négociée par le Congo et par la place prépondérante prise par celui-ci, expression chère à Patric DEVELTERE et ARISTIDE Michel. Ces auteurs renseignent que de 1961à 1968, les gouvernements successifs belges sont en quête d'une politique de coopération différente de l'administration coloniale mais sans succès. Mais en juin 1958, un nouveau gouvernement s'installe et saisit à bras le corps le problème de sous-développement. Le souci fondamental de mon département, déclare Pierre WIGNY, alors ministre belge des affaires étrangères, est d'essayer de trouver un plan concret, précis, efficace, qui mette à la disposition des pays sous-développés l'argent, les matières et les hommes qui sont nécessaires à faire tourner les machines et les plans dans lesquels doit s'insérer leur industrialisation ${ }^{4}$. C'est ici que la Belgique prendra l'option de privilégier le programme bilatéral plutôt que de participer à des nouvelles actions multilatérales. Plusieurs pas vont être franchis : la création d'une direction de l'assistance internationale au sein du département des affaires étrangères, la mise en œuvre d'un comité permanent pour la coopération internationale qui doit coordonner sur ce point les activités des différents départements, la création d'un comité des bourses, la rédaction d'un inventaire des interventions belges dans les pays en développement. Les orientations proposées reposent sur l'intérêt de toutes les parties et sur la volonté de la Belgique de dégager des fonds,sous forme de dons, pour soutenir des programmes d'aides aux pays sous-développés.

L'aide publique au développement est celle qui provient du trésor public et par conséquent celle des contribuables belges. Bien sur la population est généralement au courant par les journaux, la radio et la télévision des plus récents développements de la politique de coopération au développement : croissance du budget, remise de la dette, aide humanitaire, soutien au processus démocratique au Congo. Mais elle entend également des griefs proférés, à tort ou à raison à l'égard de l'aide publique au développement : enveloppe budgétaire dérisoire, projet inutile et dispendieux, subordination aux intérêts économiques belges. Ceci découle de la seule et unique volonté de la Belgique ou encore de ses citoyens qui sont en fait des principaux contribuables, et qui par conséquent devraient être informés de l'affectation de leurs fonds au profit de tel secteur prioritaire de développement et ce, en fonction des besoins prioritaires désignés par le partenaire(RDC). Précisons le contenu de la déclaration de Paris afin de mieux éclairer la lanterne du lecteur.

En mars 2005, plus de 100 représentants de pays donateurs (dont la Belgique), d'organisations internationales et de pays en développement avaient signé la déclaration de Paris. Cette dernière définit de quelle façon l'aide occidentale au développement peut être organisée avec une plus grande efficience dans l'optique d'accroître son impact. Elle part du pos-

4 PATRICK DEVELTERE et alii, op.cit., pp.10-11. 
tulat que l'aide accordée doit reposer sur les besoins exprimés par les pays en développement, dans ce cadre, les donateurs se baseront sur le principe d'harmonisation et d'alignement. En effet, la coopération au développement en mouvement a connu un coup d'accélération, au cours du dernier siècle. La lutte contre la pauvreté est devenue de manière univoque son objectif principal et diverses initiatives ont été prises dans l'optique d'une meilleure utilisation des moyens disponibles et de l'atteinte de résultats durables. De nouvelles formes d'aides sont développées et une attention accrue est accordée à l'efficacité et l'efficience de l'aide. Ce modus operandi influe non seulement le contenu des nouvelles interventions, mais aussi la capacité et l'attitude requises pour exécuter ce type d'intervention. La Belgique s'est officiellement engagée à appliquer le nouveau paradigme de l'aide conformément aux bonnes pratiques régissant l'aide internationale.

L'un des principaux atouts de l'aide belge, c'est son partenariat réfléchi et poussé avec les instances et organisations nationales et régionales dans le sud. L'approche participative de la Coopération Technique Belge (CTB) offre la garantie d'un dialogue véritable qui permet de consolider la durabilité des actions.

Historiquement, les relations publiques entre les deux pays sont passées de l'administration coloniale à l'assistance technique. En 1959, le Congo comptait un peu plus de 88 000 sujets belges, repartis en agents de la fonction publique et des organismes parastataux, indépendants, dirigeants et employés de sociétés privées, religieux et colons. Une ségrégation radicale régit les relations entre Blancs et Noirs, qui n'exclut d'ailleurs pas le comportement bienveillant que la majorité des blancs manifestent dans leurs rapports quotidiens et professionnels avec les congolais, attitude typiquement paternaliste. Suite aux événements des années 1958 et 1960, la composition sectorielle du personnel va subir une mutation capitale qui donnera à la coopération belge une configuration singulière : entre 1960 et 1963 , le pourcentage des enseignants passe de 20 à $60 \%$; il atteindra même $80 \%$ à la fin de la décennie, il s'agit là moins d'une volonté du gouvernement belge que d'une pénurie de professeur congolais. Cette prédominance des enseignants restera, jusqu'en 1990, ce qui est conforme au plan VAN BILLSENN qui préconisait l'indépendance du Congo dans 30ans, soit vers les années 1990. C'est l'avènement du multipartisme Congolais (zaïrois) et le début de l'éveil de conscience des congolais. Sur demande expresse des autorités congolaises et des ordres religieux, le gouvernement belge accepte de procéder à une vaste campagne de recrutement, dès les premiers mois de 1961; et pour attirer les candidats, on dispense des services militaires ceux qui s'engagent à prester trois années dans le pays(Congo) en voie de développement. Mais après cette étape historique, l'on peut s'interroger si les deux pays ont conclu sur la base d'une égalité souveraine? Si oui, cela a suscité beaucoup d'espoirs.

L'accord de Gouvernement du 01 décembre 2011 stipule ce qui suit : « En Afrique centrale, la Belgique continuera à promouvoir activement l'Etat de droit en luttant contre l'impunité, surtout concernant les violences sexuelles, la transparence dans l'exploitation des ressources naturelles sera promue au profit des populations locales. La Belgique poursuivra ses efforts en matière de soutien à la bonne gouvernance et de lutte contre la corruption. Enfin, elle encouragera la coopération régionale ». 
Sur ce continent durement touché par les conflits et la pauvreté, la Belgique s'investit pour la paix et la stabilité, le respect de la démocratie et les droits de l'homme, la bonne gouvernance, la coopération au développement et la reconstruction économique. La Belgique stimule la coopération régionale qui se concrétise grâce à l'Union Africaine et à d'autres organisations régionales ou sous régionales, et plaide en faveur de forums internationaux dans l'intérêt des pays africains. Cette position de la Belgique s'inscrit dans la droite ligne de la décentralisation prônée par les Nations Unies au bénéfice des Organisations Internationales Régionales ou Sous -régionales, qui sont les plus intéressées par le maintien de la paix et sécurité dans leur région géographique.

$\mathrm{La} \mathrm{RDC}$ et la Belgique, repartie pour des beaux jours de leurs relations, misent sur leur commission mixte de coopération. C'est dans ce cadre que les relations publiques internationales entre la Belgique et la République Démocratique du Congo se traduisent plus par la coopération dans plusieurs domaines, à savoir la Coopération technique via la CTB/ PAIDECO, la coopération scientifique entre les Universités congolaises et celles de la Belgique et qui visent la production des thèses afin de permettre au Congo de se doter d'un nombre important des professeurs et de pallier ainsi à la carence qui se constate actuellement dans ce secteur de la vie sociale du pays.

\section{Les secteurs prioritaires ciblés lors du sommet d'avril 2009}

La réunion extraordinaire du comité de partenariat élargi entre la RDC et la Belgique qui venait de se tenir à Bruxelles, avait annoncé la tenue en décembre prochain de la commission mixte belgo-congolaise pour signer le nouveau programme indicatif de coopération (PIC) entre les deux pays. La commission mixte de coopération entre la Belgique et la République Démocratique du Congo devrait se réunir encore à Bruxelles en vue de la signature d'un nouveau programme indicatif de coopération(PIC) pour la période de 2010-2013. Les ministres congolais, dont celui à la coopération Raymond TSHIBANDA et son homologue Belge Charles MICHEL, actuellement Premier ministre Belge, avaient exprimé au cours d'une conférence de presse, leur volonté de mettre en œuvre les principes arrêtés par les deux pays en avril 2009, en identifiant les secteurs prioritaires pour le développement du Congo. Les secteurs concernés sont les infrastructures, l'agriculture, l'enseignement professionnel et technique ainsi que la santé. Il ressort de cette réunion extraordinaire que la volonté de raccourcir les délais entre la désignation des projets et leurs réalisations était vraiment manifeste. Le ministre belge Charles MICHEL a exprimé la volonté de la Belgique de prendre en compte les priorités déterminées par le gouvernement Congolais et a souligné la volonté de son pays de soutenir la bonne gouvernance et la lutte contre la corruption en RDC. Et pour sa part, Raymond TSHIBANADA, chef de la délégation Congolaise, a insisté sur le profond accord entre les deux pays, sur ce qu'ils entendaient accomplir ensemble pour apporter des réponses aux problèmes rencontrés par les populations dans un pays postconflit et aux ressources financières limitées. 
D'où, a-t-il expliqué, le soin d'efficacité dans la réalisation des projets, selon le ministre congolais, les domaines prioritaires dans l'agriculture concernaient les pistes rurales, les bacs, l'accès aux intrants sans oublier la recherche agronomique, les prix des produits agricoles. Les bacs permettent le désenclavement des zones habitées et la circulation des biens et des personnes à l'intérieur du pays. Quelques bacs ont déjà été construits par la coopération belge, a-t-il rappelé.

Lors des travaux de la commission mixte de développement entre le Royaume de Belgique et la République Démocratique du Congo qui s'étaient tenus à Bruxelles les 14 et 15 décembre 2009, fut élaboré un document définissant le Programme Indicatif de coopération (PIC) bilatéral pour la période 2010-2013, ainsi que le cadre de sa mise en œuvre. Il reprend en compte les documents suivants :

- le document de stratégie de croissance et de réduction de la pauvreté (DSCRP);

- la loi congolaise sur la coopération internationale de 1982;

- la loi belge sur la coopération internationale du 25 mai 1999;

- la déclaration de Paris sur l'efficacité de l'aide et l'Agenda d'Action d'Accra sur l'efficacité de l'aide;

- La déclaration de Kinshasa;

- Le code de conduite de l'Union Européenne sur la complémentarité et la division du Travail;

- Le plan belge pour l'harmonisation et l'alignement de l'aide (2007);

- Le profil de gouvernance de l'Union Européenne;

- Les principes pour; l'engagement international dans les Etats fragiles et les situations précaires (CAD/OCDE) et

Les plans d'actions congolais et belge pour la mise en œuvre de la Résolution 1325 des Nations Unies du 31 octobre 2000 «Femmes, Paix et Sécurité »5. Un regard rapide sur ces principes dans la conclusion de cet accord bilatéral de coopération ferait penser à une parfaite égalité souveraine de deux Etats.

En outre, le principe d'appropriation des programmes de développement par la partie Congolaise, la cohérence avec le Document de stratégie de croissance et de réduction de la pauvreté (DSCRP) et ses procédures de mise en œuvre, ainsi que la synergie avec les interventions des autres partenaires au développement constituent des facteurs importants dont il faut tenir compte pour la réussite du programme ${ }^{6}$. Cette dynamique en vigueur en RDC répond largement à l'esprit des déclarations de Paris et de Rome sur l'aide extérieure ainsi qu'au consensus d'Accra auxquels la RDC et la Belgique ont souscrit.

Les objectifs du millénaire ainsi que les principes d'harmonisation, d'alignement, d'appropriation, de gestion axée sur les résultats et de responsabilité mutuelle applicable à la

5 Coopération Belgo-Congolaise, Programme Indicatif de coopération 2010-2013, Bruxelles-décembre 2009, p.2.

6 La coopération Technique Belge, op.cit., p.6, in www.ocde.org, consulté le 13 septembre 2014, vers $11 \mathrm{~h} 22^{\prime}$. 
coopération (déclaration de Paris précitée) sont systématiquement intégrés à toutes les actions. La CTB suit de près la réalité internationale et elle adapte en conséquence les règles applicables à la mise en œuvre des projets et programmes. L'expertise administrative, financière et sectorielle de la CTB est mise intégralement au service du partenaire. Les interventions se fondent sur la confiance réciproque et le développement du propre savoir-faire et ce, tant au nord qu'au sud.

Ne serait-ce que théoriquement, les deux Etats ont souhaité conclure sur la base du respect de l'égalité souveraine des Etats, principe cher au Droit International Public classique.

Les deux Etats ont souligné leur volonté de voir ce programme Indicatif de coopération servir de cadre de référence pour les autres programmes et outils de la coopération belge en RDC(Coopération indirecte, ONG locales, coopération universitaire, fonds belge de survie...) avec lesquels des synergies seront recherchées de manière systématique lors de la programmation et de la formulation des interventions.

\section{Engagements Mutuels de deux Etats}

Les parties contractantes se sont accordées sur les progrès que la RDC a enregistré depuis la tenue de la dernière commission mixte de développement (Kinshasa du 05 au 08 mars 2007). Au sortir d'une longue période de crise pendant laquelle prévalait l'aide humanitaire, la RDC est entrée dans une phase de développement structurel. La poursuite du processus de démocratisation avec la tenue d'élections locales et nationales en 2011 devrait être fondamentale. Malheureusement ces élections locales et municipales n'ont pas eu lieu jusqu'à ces jours et les députés provinciaux et sénateurs, élus en 2006, sont aujourd'hui hors mandat, y compris les gouverneurs des provinces. C'est de la sorte que le Programme Indicatif de Coopération renseigne que les pouvoirs exécutifs issus des élections continueraient à mener une politique de croissance économique et de lutte contre la pauvreté avec la mise en œuvre des cinq chantiers de la République, à savoir :

- Les infrastructures,

- La santé et l'éducation;

- Eau et électricité,

- Logement et création d'emplois.

- La Belgique, forte de sa responsabilité historique, est prête à soutenir tous ces efforts.

Tous ces deux pays étaient d'accord sur l'importance d'un bon déroulement du processus électoral, du renforcement de la société et de l'Etat dans ses fonctions de législateur et de pourvoyeur des services, de la bonne gouvernance et d'une tolérance zéro vis-à-vis de la corruption. Une gestion saine des finances publiques en conciliant les priorités du développement avec une utilisation efficace et efficiente de moyens limités était nécessaire.

Certes, il s'agit simplement d'un chapelet de bonnes intentions non suivies d'actions concrètes. La RDC est l'un des pays au monde qui possède un très bon arsenal juridique des textes de lois mais sans mesures d'encadrement efficaces et qui finissent par demeurer des lettres mortes. Les dirigeants n'ont pas la volonté politique de procéder au changement 
radical. La lutte contre la corruption est une intention bonne mais dont la matérialisation passe aussi par l'octroi au fonctionnaire congolais d'un salaire décent. Il serait absurde de lutter contre la corruption mais tout en laissant une majorité de fonctionnaires de l'enseignement supérieur et Universitaire sans Salaire de base. Certains se contentent uniquement d'une prime institutionnelle, pouvant être suspendue à tout moment.

Dans la même lancée, le processus de bancarisation initié par le gouvernement MATATA PONYO a été salué comme un atout positif de la transparence dans la gestion de la chose publique. Cependant les retombés se font attendre du fait qu'en dépit de deux contrôles biométriques, réalisés en 2013 et 2014, plusieurs membres du personnel scientifiques attendent encore se voir alignés au salaire de base.

Les deux gouvernements ont reconnu également l'intérêt de la gouvernance et de la lutte contre la corruption. Ils s'engagent pour plus de transparence et de redevabilité. Aucune offre, paiement, don ou bénéfice de quelque nature que ce soit pouvant être considéré comme un acte illégal ou de corruption, ne pourra être promis, commis, recherché, ou accepté, directement ou indirectement comme une incitation ou compensation liée aux activités dans le cadre de ce programme Indicatif de coopération, y compris toute procédure ayant trait au lancement d'attribution ou d'exécution des marchés publics.

Les deux gouvernements ont promis de s'informer mutuellement de tout incident ou suspicion d'incident de corruption liés à l'utilisation des fonds programmés dans ce PIC. En cas de non application de ces engagements, les deux parties se consulteront et détermineront les actions appropriées à prendre, qui pourraient inclure notamment le remboursement des fonds détournés et la suspension ou l'arrêt des contributions concernées.

Les parties se sont engagées au respect des droits de l'homme et de l'enfant, à l'égalité des droits et des chances des femmes et des hommes et à la pleine participation des femmes au développement. Bref, les relations publiques internationales entre les deux pays se ramènent au partage de mêmes droits reconnus aux femmes qu'aux hommes. Les appuis des partenaires à la reconstruction et à la croissance économique de la RDC n'auront pas d'impact espéré sans le développement du secteur privé. Il est dès lors primordial que soient créées les conditions favorables à son développement ${ }^{7}$. Il s'agit entre autre d'un cadre d'investissement propice (par exemple l'application du code foncier, minier, forestier, des investissements, du travail etc.), de la sécurité, de la protection juridique, de la lutte contre la corruption conformément au cadre prescrit par l'OHADA.

\section{B. La Coopération Belgo-Congolaise}

- Au regard du Programme Indicatif de Coopération de 2010-2013, la coopération belgocongolaise se réalise à travers plusieurs canaux ci-après :

- La coopération bilatérale directe (de gouvernement belge à gouvernement Congolais);

- La coopération bilatérale indirecte passant par des instances non gouvernementales;

7 Coopération Belgo-Congolaise, Agence belge de coopération au développement, Bruxelles, p.3. 
- La coopération multilatérale, développée par l'intermédiaire d'organismes internationaux tels que les agences spécialisées des Nations Unies;

- La coopération dans le cadre des accords entre Pays ACP (Afrique Caraïbe pacifique) et l'Union européenne.

Ce programme indicatif de coopération renseigne qu'en 2008, les dépenses au titre d'aide publique au Développement étaient de 122,1 millions d'euros ${ }^{8}$. Comme il s'agit d'une conclusion d'un accord bilatéral, chacune des parties avait ses prétentions que nous allons examiner séparément.

\section{Priorités de la $R D C$}

Le document de stratégie de croissance et de réduction de la pauvreté (DSCRP) de première génération définit depuis 2006 les priorités de la RDC à travers 5 piliers :

- Promouvoir la bonne gouvernance;

- Consolider la paix par le renforcement des institutions;

- Consolider la stabilité macro-économique et la croissance;

- Améliorer l'accès aux services sociaux et réduire la vulnérabilité;

- Combattre le VIH/SIDA et appuyer la dynamique communautaire?

Les outils opérationnels pour concrétiser ces cinq priorités sont entre autres :

- le Programme d'Actions Prioritaires (PAP1 2008-2009) avec pour objectifs l'atteinte du point d'achèvement,

- les cinq chantiers de la République (Education, santé, infrastructure, Electricité et Eau, Emploi et Habitat; et le contrat de gouvernance.

Eu égard aux résultats mitigés du programme d'Actions prioritaires 1 de 2008-2009, la RDC s'est dotée du programme d'Actions prioritaires 2 de 2009-2010 en attendant le cadre stratégique de lutte contre la pauvreté de deuxième génération (DSCRP2).

Le DSCRP2 allant de 2011-2015, devrait contribuer au renforcement du leadership du gouvernement congolais et des administrations sectorielles et s'articuler sur les cadres de dépenses à Moyens Termes, en sigle CDMT, national et sectoriels. Les secteurs de la santé, de l'éducation, les infrastructures, l'environnement, l'agriculture, le développement rural ainsi que l'eau et l'électricité sont considérés comme des secteurs pilotes et qui sont coulés dans le PAP2 2011-2015 en tant qu'outil opérationnel du Document de stratégie de croissance et de réduction de la pauvreté 2 . Voilà passé en revue les priorités de la RDC, véhiculées à travers sa politique extérieure dans les négociations avec le royaume de Belgique avant l'adoption de l'accord bilatéral. Il reste de parcourir également les priorités belges afin de mieux comprendre la nature de relations existantes entre ces deux nations.

8 Idem, p.4.

9 Lire le Document de Stratégie de Croissance et de Réduction de la Pauvreté de 2006. 


\section{Priorités du Royaume de Belgique en matière de coopération au développement.}

La base légale en matière de coopération internationale belge, reste la loi de 1999 portant coopération internationale belge qui limite les interventions de la coopération belge aux secteurs de la santé de base, de la formation et de l'éducation, de l'agriculture et du développement rural, des infrastructures de base et de la consolidation de la société(y compris la prévention de conflits). Les thèmes transversaux dont l'égalité des droits et des chances des femmes et des hommes, le respect de l'environnement, l'économie sociale et le respect des droits de l'enfant.

Cette loi précitée de 1999 définit plusieurs notions relatives à l'aide publique au développement, y compris celle du partenariat qu'elle présente comme étant un mode de coopération actif et participatif entre partenaires, et dans le cadre de la coopération au développement, avec une attention particulière pour le développement des capacités locales, la décentralisation des interventions au niveau des groupes-cibles visés et la responsabilisation du pays partenaire, notamment par l'association au processus de développement des pouvoirs publics, de la société civile et du secteur économique privé du pays partenaire.

C'est par cette notion de partenariat que la Belgique a ressorti dans la conclusion de ces accords, son respect à l'égard de la RDC, qu'elle considère comme Pays partenaire dans cette coopération au développement, en la responsabilisant pour des interventions au niveau des groupes -cibles visés.

L'autre document de référence reste le code de conduite de l'Union Européenne sur la complémentarité et la division du Travail qui oblige à la Belgique de savoir comment se comporter dans une relation internationale avec un Etat non membre de l'Union Européenne. Conformément à ce code, la Belgique, de commun accord avec son cocontractant Congolais, s'est engagée à concentrer ses interventions en RDC dorénavant sur trois secteurs, à savoir :

- l'agriculture;

- les pistes et les bacs,

- Et l'éducation. Il sied d'y ajouter des actions ciblées dans le domaine sanitaire.

Néanmoins, compte tenu de l'importance que revêtent le renforcement de l'Etat, de l'Etat de droit et de la lutte contre la corruption, la promotion de la bonne gouvernance constitue le centre de tous les volets du programme indicatif de coopération.

Le choix du secteur de l'agriculture a été guidé par la volonté :

- De diminuer l'insécurité alimentaire dont les populations de la RDC sont davantage victimes à cause de la volatilité des prix mondiaux des denrées de base;

- De soutenir une croissance économique équitable indispensable à la diminution de la pauvreté étant donné que cette croissance est devenue aléatoire à cause de la crise économique et financière mondiale.

Il en est de même du choix du secteur pistes et bacs couplé à celui de l'agriculture qui se justifie pour des raisons ci-après : 
- Pertinence pour la croissance économique équitable : faute de moyens d'évacuation si non à des prix de transport prohibitifs, les producteurs agricoles familiaux qui représentent la majorité des congolaises et congolais au travail se retrouvent dans une économie d'autosubsistance et n'ont aucun moyen d'enclencher un processus de création de richesses à leur profit. De plus, l'opportunité de reprise et de croissance de ce secteur est considérable.

- Pertinence pour le renforcement institutionnel : l'Etat est insuffisamment présent en milieu rural. Le manque d'accès aux services de sécurité ou à la justice empêche le rétablissement de l'Etat de droit pour la majorité de la population.

- Pertinence par rapport aux autres bailleurs de fonds : ces secteurs sont relativement peu soutenus par d'autres bailleurs mais sont aussi ceux dans lesquels s'est accumulée une grande expérience d'intervention de la coopération belgo-congolaise, que ce soit en coopération indirecte ou directe.

Pour le choix du secteur éducatif, en particulier de l'enseignement technique et de la formation professionnelle (ETFP), il est fondé sur le manque de techniciens et d'une main d'œuvre qualifiée lié aux besoins de la reconstruction et de la relance économique, de la nécessité d'apporter des chances aux nombreux jeunes désœuvrés qui constituent une source de tension sociale. Ce sous-secteur de l'éducation est par ailleurs orphelin de l'aide, l'attention des bailleurs de fonds s'étant nettement plus portées sur l'enseignement primaire.

En 2006, lors de la préparation du Programme Indicatif de coopération précédent, l'accent a été placé sur la reconstruction de l'Etat et de ses institutions. Cette stratégie reste toujours au cœur de l'approche préconisée pour la période 2010-2013. Les résultats qui ont été atteints permettent néanmoins de tabler davantage sur une approche intégrée au niveau des secteurs retenus. Ceci implique une évolution vers des programme de plus grande envergure et un suivi de l'ensemble des interventions organisées au niveau -même du secteur. La Belgique s'est déterminée à veiller à l'établissement des synergies entre les secteurs retenus et les interventions des autres acteurs de la coopérations belge.

\section{Cadre légal de la coopération belgo-congolaise.}

Les relations publiques internationales entre le Royaume de Belgique et la République démocratique du Congo, comprises comme des relations de coopération au développement entre les deux pays, sont régies par :

- La convention générale signée à Kinshasa le 27 mars 1990;

- L'accord de coopération signé à Kinshasa le 27 mars 1990;

- Le programme Indicatif de coopération 2008-2010 signé en mars 2007 entre le Royaume de Belgique et la République Démocratique du Congo;

- Le programme Indicatif de coopération2010-2013 entre le Royaume de Belgique et la République Démocratique du Congo. 
La Belgique pose des actes dans le domaine de la coopération parce qu'elle se fonde sur sa loi du 25 mai 1999 portant loi relative à la coopération internationale belge ${ }^{10}$ et qui détermine son cadre d'action et son étendue. C'est cette loi, en son article 13 qui précise ce qu'il faut retenir au sujet de l'aide publique au développement. De la sorte, elle précise que la coopération internationale belge a pour objectif prioritaire le développement humain durable, à réaliser par le biais de la lutte contre la pauvreté sur la base du concept de partenariat et dans le respect des critères de pertinence pour le développement ${ }^{11}$. Tous les principes qui sont repris parmi les priorités de la Belgique sont prédéfinis dans cette loi qui passe pour l'instrument juridique belge de référence en matière de coopération au développement.

La coopération technique belge $(\mathrm{CTB})$ et le secrétariat Général à la coopération internationale sont responsables de la mise en œuvre du programme Indicatif de coopération.

Le statut juridique de la CTB en République Démocratique du Congo est réglé par l'échange de Lettres des 20 octobre et 2 décembre 2009 entre l'Ambassade du Royaume de Belgique à Kinshasa et le Ministre de la coopération internationale et Régionale de la RDC. La CTB est l'instrument de mise en œuvre de la politique belge d'aide au développement. Elle est prestataire des services publics, elle soutient pour le gouvernement fédéral belge et plus précisément, pour le service public fédéral des Affaires étrangères, du commerce extérieur et de la coopération au développement, les pays en développement dans leur lutte contre la pauvreté. Grace à son expertise de terrain, la CTB exécute également des prestations pour le compte d'autres organisations nationales et internationales contribuant à un développement humain durable ${ }^{12}$. Elle est opérationnelle depuis 1999 et gère plus de 230 projets répartis dans 25 pays en Afrique, Asie et Amérique latine. Elle est plus présente en Afrique et plus spécialement au Congo, à cause du retard qu'accuse l'Afrique pour décoller vers un développement durable. Ces pays sont régulièrement déchirés par des guerres et des conflits internes qui les empêchent de connaitre leur expansion.

Par ailleurs, si en Droit International, on a adopté l'appellation « Pays en développement », c'est pour éviter cette connotation péjorative que véhiculent les concepts de «pays sous-développés ». Il me semble que la RDC ne soit même pas digne d'être classée parmi les pays en développement, car rien ne permet qu'elle de prétendre qu'elle se soit engagée dans un processus de développement. Au contraire, elle connait du recul en dépit de cette coopération au développement.

La nature de tous ces actes d'engagement pris dans cet accord bilatéral, semble poser quelques petits problèmes. Théoriquement, tous ces documents incluent les priorités de ces deux contractants dans la mesure où rien ne permet d'affirmer qu'il s'agirait des accords bilatéraux déséquilibrés. Il s'agit d'un partenariat au sein duquel l'un réalise des dons pro-

10 Loi relative à la coopération internationale belge, publiée par le moniteur belge, Bruxelles, le 25 mai 1999.

11 Lire l'article3, al.1.Titre II de la loi du 25 mai 1999 précitée.

12 Coopération Technique Belge, Agence belge de coopération au développement, Bruxelles, p.2, info@btcctb.org, WWW.BTCCTB.ORG, consulté le 23 décembre 2014, vers 11h25'. 
venant des contribuables belges en vue susciter la croissance de l'autre. Mais vu le retard qu'accuse la RDC, rien ne permet de croire cette coopération belge porte des fruits escomptés du fait d'abord du manque de volonté politique de l'exécutif congolais qui ne semble pas prêt à provoquer un changement radical. Lorsqu'on parle de la tolérance zéro dans le programme d'actions de la RDC, que fait la Belgique concrètement pour pousser son cocontractant Congolais à passer réellement à l'acte. Ce sont les mêmes personnes qui détournent les fonds au sommet et qui sont récompensées en se retrouvant toujours au sein de l'exécutif. En clair, la nature des différents textes qui régissent cette coopération belgocongolaise ne pose pas de problème en elle-même, mais plutôt les animateurs qui ne sont fiables.

La CTB en venant au Congo, a commencé par s'installer en milieu urbain comme la ville de Lubumbashi. Elle a concentré son action de développement sur l'unique commune Annexe où elle a réfectionné le bâtiment communal, en construisant plusieurs écoles en périphérie de la ville de Lubumbashi et plusieurs bornes fontaines pour enfin quitter la ville vers le milieu rural. En fait, la coopération belgo-congolaise étant l'une des plus importantes coopérations bilatérales en RDC, elle s'est concentré sur un nombre spécifique de zones géographique et a créé des économies d'échelle. De la sorte, les parties belge et congolaise s'engageaient à mettre en place et maintenir une tolérance zéro vis-à-vis de l'application stricte de la législation en matière de protection et de sécurité routière, afin de prévenir des dégâts aux travaux exécutés (tel est le cas du respect des limites de charge et de barrières de pluie). En milieu rural, la CTB est entrain de réfectionner plusieurs petits ponts de desserte agricoles pour désenclaver ces villages.

La partie congolaise s'était dite d'accord pour veiller à la mise à disposition locale des ressources humaines suffisantes et de qualité, surtout au sein de la Direction des Voies de Desserte Agricoles, en sigle DVDA. Il a été décidé également qu'un appui serait donné aux comités locaux d'entretien des Routes (CLER) et aux comités de Lutte Anti Tracasserie(CLAT) afin de renforcer la surveillance et la dénonciation des contraventions à la DVDA, la police nationale et les autorités locales. Dans le programme indicatif de coopération, l'on annonçait la mise en œuvre des mesures mitigeant les effets secondaires du désenclavement, surtout concernant l'exploitation illicite des ressources naturelles. Les bénéficiaires directs sont des exploitants familiaux actuellement enclavés dans les zones d'intervention et indirectement, ce sont les populations en générale qui vivent dans les zones d'intervention et les centre de consommation.

Encore une fois, un accent est mis sur la gouvernance afin que tous ces objectifs ne restent pas des simples chapelets d'intentions. Et compte tenu de l'importance que revêt la gouvernance pour le développement durable et l'efficacité de l'aide, le renforcement de la capacité des institutions publiques dans les secteurs de concentration devenait un objectif central. Les principes de gouvernance (transparence, participation, redevabilité, inclusion etc.) devraient figurer au programme sectoriel et la gestion des interventions.

A cet effet, les dossiers techniques et financiers (DTF) comprendront une analyse solide des éléments de la gouvernance dans les secteurs de concentration, y compris des capacités 
des acteurs concernés, de façon à les renforcer, et des effets négatifs potentiels sur la dynamique existante dans chaque secteur.

Les relations internationales évoluent quelque fois dans une sociologie des conflits. On se rappelle à ce propos qu'entre la RD Congo et le Royaume de Belgique, le ciel n'a pas toujours été clair. A une certaine époque, ces relations ont été talonnées de contentieux qui ont forcé la rupture de la coopération. Après des années de brouille, la RD Congo et le Royaume de Belgique ont harmonisé leurs relations de coopération bilatérale.

Raymond TSHIBANDA précise que cette coopération bilatérale redynamisée jette les bases d'une ère porteuse d'espoir, fondée sur le respect mutuel, la conscience de l'importance accordée à ce processus et la volonté d'inscrire ces relations dans la durée tout en gardant l'esprit de la déclaration de Paris. Selon le ministre congolais de la Coopération internationale et -régionale, cette coopération ré- dynamisée a amené à coprésider la réunion du comité des partenaires qui avait donné un coup de relance aux relations belgo-congolaises $^{13}:$ «On s'est engagé à travailler pour renforcer l'efficacité des projets concernés », a renchéri le ministre rd congolais de la Coopération internationale et régionale. Et de poursuivre « coté congolais, un atelier à ce sujet avait réuni différents acteurs de la société civile et les ministères techniques par souci de concentration sur des actions prioritaires et d'identification des besoins des populations congolaises ». Quatre secteurs ont été ciblés. Il s'agit entre autres, de l'agriculture, du développement rural, des pistes rurales, des bacs, de l'enseignement technique et professionnel ainsi que des actions ciblées dans le secteur de la santé. Si le gouvernement de la RDC a proposé ses priorités, c'était à l'issue des concertations avec différentes couches de la population congolaise. Encore une fois, elles reflètent les besoins réels et prioritaires de la population congolaise mais peuvent -ils s'avérer réalisables sans une volonté politique manifeste des leaders congolais.

Les propos du ministre Congolais à la coopération confirment la nature juridique de cet accord de partenariat belgo-congolais, fondé sur le respect mutuel d'égalité souveraine reconnue à chaque Etat. Si par le passé, la Belgique pouvait s'engager dans des relations publiques inégalitaires avec la RDC du fait de sa position stratégique au sein de l'Union européenne et dès par les liens historiques de la colonisation, dorénavant elle entre dans une relation redynamisée et fondée sur l'égalité souveraine. Cependant, comme la main du donateur est toujours au-dessus de celle du bénéficiaire, la Belgique semble toujours posséder une longueur d'avance par rapport au Congo, qui lui ne fait que recevoir. Toutefois, quand elle fait des dons dans le cadre de l'aide publique, elle détermine les conditions pour bénéficier de cette assistance destinée à favoriser un développement rapide et durable du Congo. Par conséquent, la Belgique se réserve toujours un droit de contrôle qui ne peut aucunement s'entendre comme étant une immixtion dans les affaires intérieures du Congo.

13 MILOR, Accord bilatéral RDC- Belgique, Forum des AS, dernière édition du 06/10/2009, in lettre@digitalcongo.net, relapub@digitalcongo.net, consulté le 19 décembre 2014 vers 10h43'. 


\section{CONCLUSION}

Pour clore cette étude relative à l'encadrement juridique des relations publiques internationales entre le Royaume de Belgique et la République du Congo, il sied de rappeler que dès par les liens historiques, ces deux pays entretiennent des relations très soudées et même fiables. Vu que l'indépendance du Congo avait été mal négociée et accordée par la métropole, cette dernière se reproche toujours de quelque chose et se sent obligé d'intervenir à tout moment afin d'aider au développement de son ancienne colonie. Celle-ci accuse un retard considérable dans son développement. C'est de la sorte que la Belgique a mis en place un système d'aide au développement à travers lequel elle donne priorité à l'aide bilatérale directe ou indirecte.

Par ailleurs, ces deux sont arrivés à la conclusion de plusieurs accords bilatéraux portant sur des secteurs divers et ce sur la base du respect mutuel, comme a eu, à le témoigner, Raymond TSHIBANDA, alors ministre Congolais de la coopération et chef de la délégation congolaise à ce sommet de Bruxelles. Dans l'entre temps, Charles MICHEL, alors chef de la délégation belge affirmait devoir respecter les priorités désignées par les experts congolais. Bref, théoriquement, il est permis sous réserve d'affirmer que les deux pays ont vraiment négocier dans le respect mutuel et réciproque. Cependant, pourquoi, en dépit de tous ces accords bilatéraux portant (au moins 8 accords), la RDC prend du temps pour décoller? Nous avons passé en revue tout un chapelet de plusieurs bonnes intentions dont la lutte contre la corruption, la lutte contre la pauvreté... Point n'est besoin de s'engager dans des relations internationales si il n'existe aucun intérêt. Ne dit-on pas que les relations interétatiques sont des relations de puissance et que seuls les intérêts des Etats en présence, guident leurs actions.

Nous estimons en guise de recommandations que les relations Publiques internationales entre la RDC et la Belgique ne souffrent pas du déficit de leurs négociations mais bien au contraire du manque des bons animateurs. Il ne suffit pas de fustiger la corruption et de déterminer la sanction qu'encoure celui qui transgresse, mais il convient de sanctionner directement ceux qui s'y adonnent. Cependant du côté du co-contractant congolais, l'on avance a contrario parfois en cherchant à récompenser ceux qui détournent les deniers publics ou bien, c'est le règne de l'impunité. Dans cette hypothèse, faut- il que lorsqu'un haut responsable belge fustige une corruption généralisée au sein des institutions congolaises, que des bouches se lèvent pour le taxer d'immixtion dans les affaires intérieures du Congo. Le changement doit être total et radical afin que la RDC gagne du crédit dans la conduite de sa politique extérieure vis -à-vis des Etats du Nord.

\section{BIBLIOGRAPHIE}

1. Loi relative à la coopération internationale belge du 25 mai 1999.

2. Document de stratégie de croissance et de réduction de la pauvreté de 2006, 2006 
3. Coopération belgo-congolaise, Programme Indicatif de coopération2010-2013, Bruxelles, 21 décembre 2009

4. La coopération Technique Belge, agence belge de coopération au développement, Bruxelles,

5. MILOR, "Accord bilatéral RDC-Belgique », in Forum des As

6. PATRICK DEVELTERE et Aristide MICHEL, Chronique d'un demi-siècle de coopération belge au développement, service de communication, Bruxelles

\section{WEBOGRAPHIE}

1.1ettre@digitalcongo.net

2.relapub@digitalcongo.net

3. Info@btcctb.org

4. www.BTCCTB.ORG

5. www.ocde.org

6. www.diplomatie.be

7. www.dg-d.be 